\title{
Enhanced transmission of optically thick metallic films at infrared wavelengths
}

\author{
Dezhuan Han, Xin Li, Fengqin Wu, and Jian Z: \\ Surface Physics Laboratory and Department of Physics, \\ Fudan University, Shanghai 200433, People's Republic of China
}

(Dated: April 10, 2018)

\begin{abstract}
For an optically thick metallic film, the transmission for both $s$ - and $p$-polarized waves is extremely low. If the metallic film is coated on both sides with a finite dielectric layer, light transmission for $p$-polarized waves can be enhanced considerably. This enhancement is not related to surface plasmon-polaritions. Instead, it is due to the interplay between Fabry-Perot interference in the coated dielectric layer and the existence of the Brewster angle at the dielectric/metallic interface. It is shown that the coated metallic films can act as excellent polarizers at infrared wavelengths.
\end{abstract}

PACS numbers: 42.25.Bs, 48.47.De, 42.25.Hz, 42.79. Ci

Metal surfaces are highly reflective over a very wide range of wavelengths. This is the reason why metals are commonly used as mirrors in our daily life and in optical technologies as well. It is known that the transmission of an optically thick metallic film is very low for wavelengths in the visible range or below. However, for $p$-polarized waves transmission could be very high when incident light excites the coupled surface plasmon-polaritons (SPPs) on both sides of the metallic film. ${ }^{\underline{1}}$ SPPs are a kind of electromagnetic excitations existing at the interface between a metal and a dielectric medium ${ }^{2}$ For a flat metallic surface, $p$-polarized incident waves cannot directly excite SPPs owing to the wavevector mismatch between incident waves and SPPs. Normally, an attenuated total reflection technique is adopted to generate evanescent waves which can excite SPPs $\underset{2}{2}$ Extraordinary transmission has been also found for a metallic film perforated with subwavelength hole arrays $\mathrm{s}^{3}$ or slits $\underline{\underline{4}}$ It is believed that the excitation and coupling of SPPs on both surfaces of the metallic film play an important role in such an extraordinary transmission.

In this letter, we report theoretically an enhanced transmission of $p$-polarized waves at infrared wavelengths for an optically thick metallic film coated with a dielectric layer on both sides. It is found that the enhanced transmission is not due to SPPs. Instead, it relies on the interplay between Fabry-Perot interference in the dielectric layer and the existence of a Brewster angle window at the dielectric/metallic interface.

In our numerical simulations, without loss of generality, metallic films are assumed to be Ag. The dielectric constant of $\mathrm{Ag}$ is described by the Drude model

$$
\varepsilon(\omega)=1-\frac{\omega_{p}^{2}}{\omega^{2}+i \gamma \omega},
$$

where $\omega_{p}$ is the plasma frequency and $\gamma$ is the parameter related to the energy loss. For Ag, the parameters used are $\omega_{p}=1.15 \times 10^{16} \mathrm{rad} / \mathrm{s}$ and $\gamma=9.81 \times 10^{13} \mathrm{rad} / \mathrm{s}$, which are obtained by fitting to the experimental data ${ }^{5}$ at near and mid infrared wavelengths.

At a dielectric/dielectric interface, there exists a Brewster angle, at which the reflectance for $p$-polarized waves is zero. To show the fact that a metallic surface also possesses a Brewster angle, the reflectance spectra for $p$ polarized waves incident from a dielectric medium with a refractive index of 1.5 upon a $\mathrm{Ag}$ surface at different incident angles are shown in Fig. 1] For s-polarized waves, the reflection at the Ag surface is very high and increases monotonically with increasing incident angle. For $p$-polarized waves, however, there exist dips in the reflectance spectra for incident angles near $90^{\circ}$. The positions of the dips can be viewed as the Brewster angle at the Ag surface. Unlike the dielectric surface, reflectance at the metallic surface is nonzero at the Brewster angle. At short wavelengths (visible or near infrared), dips in the reflectance spectra are rather shallow so that the Brewster angle is not well-defined. Dips become sharper with the increase in wavelength. The Brewster angle is wavelength dependent and shifts upward with the increasing wavelength. It approaches $90^{\circ}$ at the long wavelength limit. For a dielectric/dielectric interface, the Brewster angle is given by $\tan \theta_{B}=n_{2} / n_{1}$, where $n_{1}$ and $n_{2}$ are the refractive indices of two dielectric media. For the dielectric/metallic interface, the Brewster

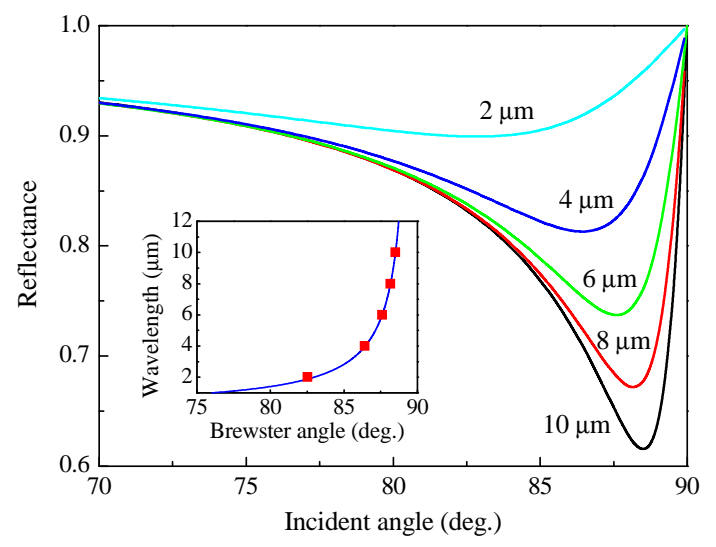

FIG. 1: (color online). Reflectance spectra of $p$-polarized waves incident from a dielectric medium (refractive index 1.5) upon a $\mathrm{Ag}$ surface at different incident angles. Labels indicate different wavelengths. Inset shows the calculated Brewster angles from Eq. (2) and those obtained from the reflectance spectra. 


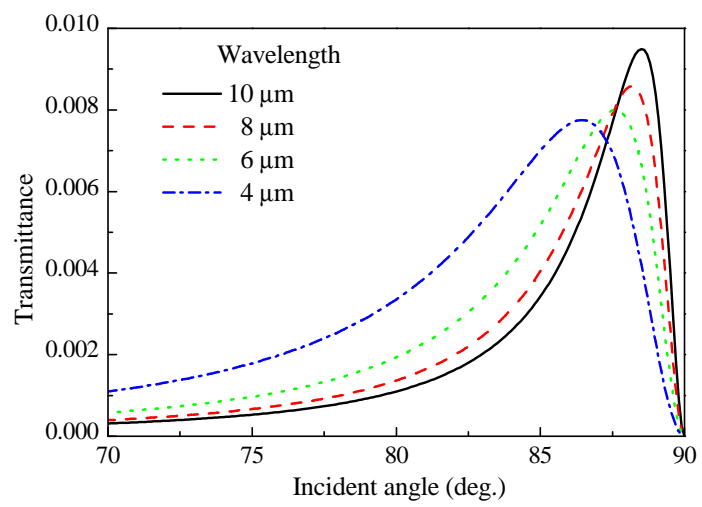

FIG. 2: (color online). Transmittance spectra of $p$-polarized waves as for a $80 \mathrm{~nm} \mathrm{Ag}$ film immersed in a dielectric medium (refractive index 1.5) at different incident angles.

angle cannot be given by a simple formula $\underline{\underline{6}}$ However, for wavelengths considered here it is found that the Brewster angle at the dielectric/metallic interface can be well approximated by the following relation

$$
\tan \theta_{B}=\left(\frac{\left|\varepsilon_{2}\right|}{\varepsilon_{1}}\right)^{1 / 2}
$$

where $\varepsilon_{1}$ and $\varepsilon_{2}$ are the dielectric constants of the dielectric and metallic media, respectively. In Fig. 11 Brewster angles at different wavelengths obtained from the above relation and from the reflectance spectra are also given for comparison. It can be found that Brewster angles predicted by Eq. (2) agree well with those obtained from the reflectance spectra.

The existence of the Brewster angle at a metallic surface can manifest a higher transmission of $p$-polarized waves for a metallic film at the Brewster incident angle with respect to other incident angles. Figure 2 shows the transmittance spectra of $p$-polarized waves for a 80 $\mathrm{nm} \mathrm{Ag}$ film immersed in a dielectric medium at different incident angles. For $s$-polarized waves, transmission is extremely low for all incident angles. Thus, the 80 $\mathrm{nm} \mathrm{Ag}$ film is optically thick enough to block $s$-polarized waves. For $p$-polarized waves, the situation, however, is a bit different. The overall transmission is still very low, especially for incident angles not close to grazing angles. It is interesting to note that there exist some maxima in the transmittance spectra at some grazing incident angles close to $90^{\circ}$. For a fixed wavelength transmittance increases monotonically for incident angles varying from normal incidence, reaches a maximum at a certain grazing incident angle, and then decreases monotonically up to the $90^{\circ}$ incident angle. It is obvious that the positions of the transmission peaks in Fig. [2] coincide well with the Brewster angles at the dielectric/metallic interface.

We now consider a Ag film coated with a dielectric layer symmetrically on both sides. The refractive index of the coated dielectric layer is taken to be 1.5. From the above discussions it is known that the Brewster angle is close to $90^{\circ}$. If $p$-polarized waves are incident from air upon the dielectric layer, the refracted angle may be smaller than the Brewster angle at the dielectric/metallic interface since the dielectric constant of air is smaller than that of the dielectric medium. As a result, we may not access the Brewster angle at the dielectric/metallic interface. To exclude this possibility, two prisms with a higher refractive index than the coated dielectric layer are introduced to situate on both coated dielectric layers. Practically, it is not an easy job to get grazing incidence close to $90^{\circ}$. The introduction of prisms, however, can overcome this difficulty. This can be easily seen from Snell's law

$$
\sin \theta_{1} / \sin \theta_{2}=n_{d} / n_{p}
$$

where $n_{p}$ and $n_{d}$ are the refractive indices of the prism and the coated dielectric layer, respectively; $\theta_{1}$ is the incident angle in the prism; $\theta_{2}$ is the refracted angle in the dielectric layer, also the incident angle upon the metallic film. Without loss of generality, the refractive index of prisms is chosen to be 3 . Thus, the critical angle at the prism/dielectric interface is $30^{\circ}$. For $\theta_{1}$ larger than this critical angle, evanescent waves will be generated. This configuration has been commonly used to excite SPPs ${ }^{2}$

In the coated layer, Fabry-Perot interference is expected owing to the multiple reflection between the prism/dielectric and dielectric/metallic interfaces. When traversing across the coated dielectric layer, two successively transmitted waves have a phase difference

$$
\delta=\frac{2 \pi}{\lambda_{0}} 2 n_{d} d \cos \theta_{2}+\phi,
$$

where $d$ is the thickness of the coated dielectric layer, $\lambda_{0}$ is the wavelength in vacuum, and $\phi$ is an additional phase shift due to the metallic surface. Transmission into the metallic film can be enhanced if $\delta=(2 m+1) \pi$, where the integer $m$ takes the value of $0,1,2, \ldots$ Combining

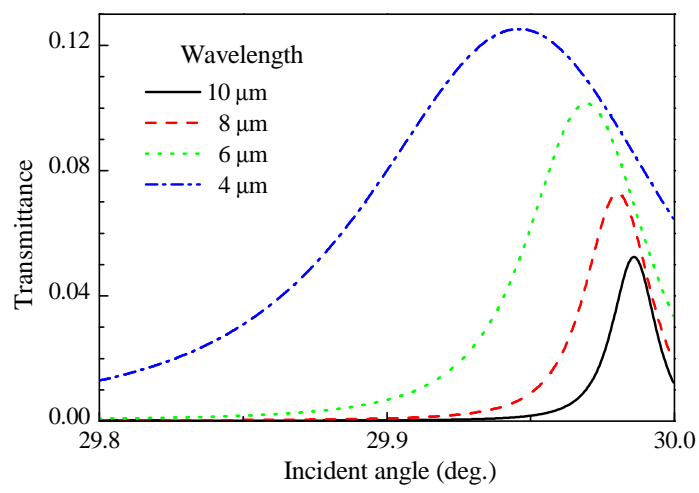

FIG. 3: (color online). Transmittance spectra of $p$-polarized waves for a coated $\mathrm{Ag}$ film with a thickness of $80 \mathrm{~nm}$ at different incident angles from the prism. Labels indicate different wavelengths. The thickness of the coated dielectric layer is determined from Eq. (5). The refractive indices of the coated dielectric and the prism are 1.5 and 3 , respectively. 


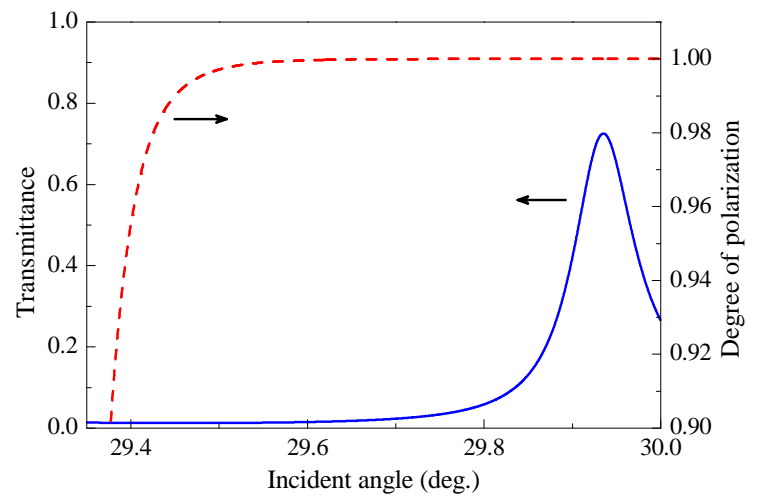

FIG. 4: (color online). Transmittance spectrum of a $p$ polarized wave of $\lambda_{0}=4 \mu \mathrm{m}$ and degree of polarization for a coated Ag film with a thickness of $40 \mathrm{~nm}$ as a function of the incident angles from the prism. The thickness of the dielectric layer is $6.45 \mu \mathrm{m}$.

this effect with the Brewster angle window, enhanced transmission should be expected.

In Fig. 3 transmittance spectra for a coated $80 \mathrm{~nm}$ Ag film situated between two prisms at difference incident angles are shown. To get enhanced transmission the choice of the thickness of the coated dielectric layer is crucial. At the Brewster angle, it is found that the additional phase difference due to the metallic surface is $\pi / 2$. Thus, the minimal thickness of the coated dielectric layer that renders an enhanced transmission is determined from

$$
d=\frac{\lambda_{0}}{8 n_{d} \cos \theta_{B}}
$$

It is obvious from Fig. 3 that transmission is largely enhanced with respect to the same metallic film situated in a dielectric medium. The enhanced factor of the transmission peak for the wavelength of $4 \mu \mathrm{m}$ is over 16 and that for the wavelength of $10 \mu \mathrm{m}$ is more than 5 . It should be noted that transmission is also dependent on the thickness of the metallic film. Higher transmission can be obtained if we reduce the thickness of the metallic film. For smaller thickness of the metallic film, the minimal thickness of the coated dielectric layer that renders enhanced transmission may deviate somewhat from that obtained from Eq. (5) owing to the coupling of the two dielectric/metallic interfaces.
As shown above, $p$-polarized waves possess a high transmission for a coated metallic film, while $s$-polarized waves have an extremely low transmission. This feature can render infrared polarizers with excellent performance possible. To obtain a good performance, the thickness of the coated dielectric layer and the metallic film should be chosen properly. A good polarizer is characterized by a high transmission and a high degree of polarization, defined by

$$
P=\frac{T_{p}-T_{s}}{T_{p}+T_{s}}
$$

where $T_{p}$ and $T_{s}$ are the transmittance of $p$ - and $s$ polarized waves, respectively. For a perfect polarizer, the degree of polarization should be 1 . In Fig. 团 the transmittance spectrum of a $p$-polarized wave with $\lambda_{0}=4 \mu \mathrm{m}$ for a coated $\mathrm{Ag}$ film with a thickness of $40 \mathrm{~nm}$ and the degree of polarization of the system are shown. A thickness of $6.45 \mu \mathrm{m}$ for the dielectric layer is chosen in order to obtain a maximal transmission at certain incident angle. It is obvious that this polarizer has a high transmittance (over 70\%) for the $p$-polarized wave around the incident angle of $29.93^{\circ}$ from the prism, while its degree of polarization is nearly perfect.

In the visible range, conventional polarizers have excellent performance and are easily attainable. In the infrared regime, on the contrary, the conventionally used metallic wire-type polarizers are costly and their performance is less satisfactory with respect to those in the visible range ${ }^{7}$ Our results indicate that the coated metallic film could act as an excellent polarizer at infrared wavelengths. The results shown in Fig. 4 are for $\lambda_{0}=4 \mu \mathrm{m}$. For other infrared wavelengths, we can also obtain satisfactory performance for a coated metallic film provided that the thicknesses of the coated dielectric layer and the metallic film are properly chosen.

In summary, we studied theoretically the transmission of optically thick metallic films. It was shown that there exists a Brewster angle window at the dielectric/metallic interface. Incorporated with the Fabry-Perot interference, a coated metallic film can have a largely enhanced transmission. This feature can render excellent polarizers at infrared wavelengths possible.

This work was supported by CNKBRSF, NSFC, PCSIRT, and Shanghai Science and Technology Commission, China. We thank Dr. S. Meyer for a critical reading of the manuscript.
* Electronic address: jzi@fudan.edu.cn

1 R. Dragila, B. Luther-Davies, and S. Vukovic, Phys. Rev. Lett. 55, 1117 (1985).

${ }^{2}$ H. Raether, Surface Plasmons (Springer-Verlag, Berlin, 1988).

3 T. W. Ebbesen, H. J. Lezec, H. F. Ghaemi, T. Thio, and P. A. Wolff, Nature (London) 391, 667 (1998).

4 J. A. Porto, F. J. García-Vidal, and J. B. Pendry, Phys.
Rev. Lett. 83, 2845 (1999).

${ }^{5}$ Handbook of Optical Constants of Solids, edited by E. D. Palik (Academic Press, New York, 1985).

${ }^{6}$ D. Bedeaux and J. Vlieger, Optical Properties of Surfaces (Imperial College Press, London, 2001), p. 64.

7 J. M. Bennett and H. E. Bennett, Handbook of Optics, Ed. by W. Driscoll (McGraw-Hill, New York, 1978). 